\title{
Preoperative cardiopulmonary exercise testing in England - a national survey
}

\author{
Sam Huddart ${ }^{\text {* }}$, Emily L Young ${ }^{1}$, Rebecca-Lea Smith², Peter JE Holt ${ }^{3,4}$ and Pradeep K Prabhu ${ }^{1}$
}

\begin{abstract}
Background: Cardiopulmonary exercise testing (CPET) has become well established in the preoperative assessment of patients presenting for major surgery in the United Kingdom. There is evidence supporting its use in riskstratifying patients prior to major high-risk surgical procedures.

We set out to establish how CPET services in England have developed since the only survey on this subject was undertaken in 2008 (J Intensive Care Soc 2009, 10:275-278).

Methods: Availability of preoperative CPET and contact details were collected via a telephone survey and email invites to complete the online survey were sent to all contacts. The survey was live during March and April 2011.

Results: We received $123(74 \%)$ responses from the 166 emails that were sent out. In total, 32\% (53/166) of all adult anesthetic departments in England have access to preoperative CPET services and a further 4\% (6) were in the process of setting up services. The number of departments offering preoperative CPET, including those in the process of setting up services, has risen from 42 in 2008 to 59 in 2011, a rise of over 40\%. Only 61\% of the clinics are run by anesthetists and $39 \%$ of clinics have trained cardiorespiratory technicians assisting in the performance of the test. Most of the clinics (55\%) rely solely on a bicycle ergometer. Vascular surgical patients are the largest group of patients tested, and the majority of tests are run to a symptom-limited maximum. We estimate that 15,000 tests are performed annually for preoperative assessment in England. Only 37\% of respondents were confident that the tests performed were being billed for.
\end{abstract}

Conclusions: CPET is increasing in popularity as a preoperative risk assessment tool. There remains a lack of consistency in the way tests are reported and utilized. The results highlight the extent and diversity of the use of preoperative CPET and the potential for further research into its use in unstudied patient groups.

Keywords: Cardiopulmonary exercise testing, Exercise testing, Preoperative assessment, Preoperative risk assessment, National survey

\section{Background}

Cardiopulmonary exercise testing (CPET) has become well established in the preoperative assessment of patients presenting for major surgery in the United Kingdom. There is some evidence to support the use of CPET-derived variables for risk stratification and allocation to an appropriate level of post-operative care in many major surgical specialties [1-18], although there are no published randomized control trials. To date there has been one published survey into the use of preoperative cardiopulmonary exercise testing (PCPET) in England (performed in 2008, published

\footnotetext{
* Correspondence: samhuddart@nhs.net

${ }^{1}$ Department of Anaesthesia, Royal Surrey County Hospital, Guildford GU2 7XX, UK

Full list of author information is available at the end of the article
}

in 2009) [19]. This survey identified 30 units in England that provided PCPET services for preoperative assessment, and a further 12 units in the process of setting up a service. We set out to determine how PCPET services have progressed since the 2008 survey and to ascertain the wider interest in PCPET in England.

\section{Methods}

The survey was conducted in two main stages. The first stage involved telephoning anesthetic secretaries in every NHS trust in England. This way, we hoped to find out how many departments performed PCPET, and to obtain contact details for a named consultant responsible for the service. The second stage involved sending a link to

\section{Biomed Central}


an online survey to each of these contacts. The National Research Ethics Service (NRES) has confirmed that, in accordance with their guidelines, the survey does not require formal ethical approval.

\section{Telephone survey}

We accessed the NHS England website in December 2010 [20]. This website lists details of all 168 NHS trusts in England, 159 of which provide surgical services for adult patients. We contacted these trusts by telephone and identified 166 functionally separate anesthesia departments providing services for surgical procedures in adults. We contacted the anesthetic secretaries for all of these departments by telephone asking if preoperative CPET is available in their department and for contact details of the clinician responsible for either CPET or preoperative assessment.

\section{Online survey}

We composed the survey using an online survey tool (http://www.surveymonkey.com). For the majority of questions, we used a multiple-choice format with an additional free-text option for comments. Not all questions were compulsory for submission of the completed survey. The survey questions and structure are shown in Additional files 1 and 2.

The survey was sent to each identified email contact as a link within the email. A maximum of three reminder emails were sent over the following four weeks. The survey was live in March and April 2011.

\section{Repeat telephone survey}

Some of the secretaries contacted in the original telephone survey did not know if their department had access to PCPET. If we did not receive a response to the online survey and were originally unsure if they had PCPET services, we re-contacted them by telephone, so that we could confirm the total number of departments in England with PCPET services.

\section{Results}

We contacted all 166 (100\%) anesthesia departments by telephone. In total (after the repeat telephone survey described above) we identified 53 departments who offer PCPET (32\%).

\section{Online survey}

We received a total of 128 responses to the survey. Five of these were duplicate responses from individuals in the same department. Only the first response received from each of these departments was included in the analysis. As such our overall response rate was 74\% (123/166).

We received $49(40 \%$ of total) responses from departments that offer PCPET and 74 (60\% of total) responses from departments without access to PCPET services.

\section{Departments without PCPET services $(n=74)$}

Thirty-five (47\%) of those who responded have made an attempt to set up PCPET services that was unsuccessful. The reasons given for failed attempts included: financial (43\%), perceived lack of clinical need (11\%) and insufficient evidence of benefit (6\%).

Thirty-three departments (45\%) have not attempted to set up a service. Reasons stated for this included: financial constraints or lack of resources (39\%), inappropriate case mix (9\%), training issues (3\%), lack of support from other departments $(3 \%)$ and conflicting evidence for clinical benefit (3\%).

Six departments $(8 \%)$ are in the process of setting up a peri-operative CPET service.

The survey responses rates are summarized in Figure 1.

\section{Departments with PCPET services $(n=49)$}

The majority of respondents to the survey were anesthetists (90\%), the remainder being clinical scientists (6\%) and physicians (4\%).

\section{Logistical aspects of PCPET services}

Forty-five respondents (92\%) indicated that testing is performed in-house. One department refers patients to a private CPET clinic as well as testing patients in-house themselves.

The majority of tests are conducted by anesthetists. Some are conducted by a variety of other clinicians and nonclinicians (Figure 2). Three respondents do not have any assistance during testing (6\%) and 19 (39\%) are assisted by a trained cardio-respiratory technician. Other assistance during testing includes: operating department practitioners (14\%), pre-assessment nurse (14\%), nursing auxiliary (6\%), anesthetic practitioner (2\%), physicians' assistants in anesthesia (2\%) and research nursing staff (2\%).

Clinics are operated in a variety of locations: in the preassessment clinic (39\%), in the respiratory laboratory/clinic (27\%), in the cardiology department (12\%), in a ward area (4\%), in the outpatients department $(4 \%)$, in a research laboratory (2\%) and on the intensive care unit (2\%). The responsibility for maintenance, cleaning and sterilization of reusables and stocking of disposables lies with the clinician (20\%), technician $(49 \%)$ or a nurse $(10 \%)$.

\section{Clinical aspects of PCPET services}

Referrals for CPET are received from multiple sources. Departments receive referrals from surgical colleagues (76\%), anesthetic colleagues (69\%), the pre-assessment clinic (67\%) and from multi-disciplinary team meetings (35\%). Interestingly only $22 \%$ of departments include 


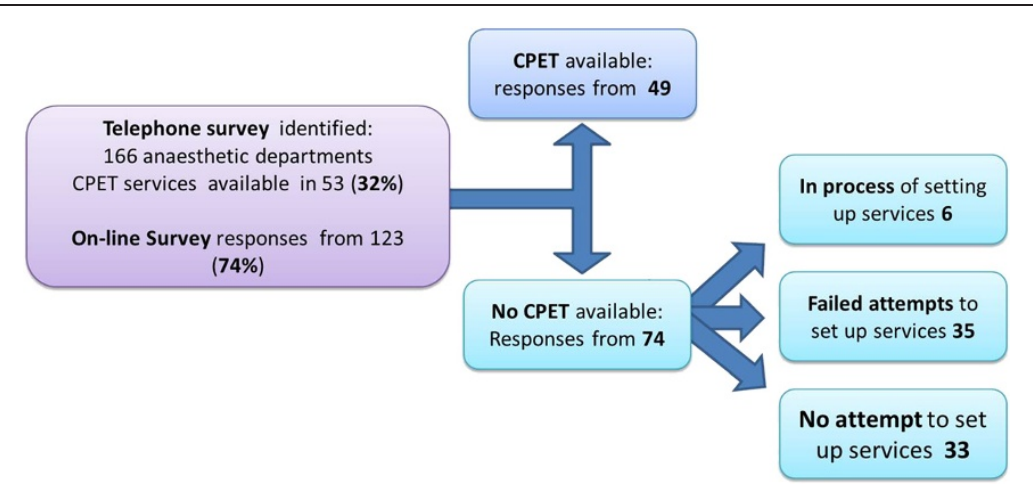

Figure 1 Survey response rates.

strict predetermined criteria as a part of their referral pathway. Of the total, $8 \%$ reported receiving referrals from cardiologists and respiratory physicians.

Twenty-four (49\%) departments consent their patients verbally for PCPET, 11 (22\%) require formal written consent and seven (14\%) do not consent their patients prior to testing. This is a significant deviation from the practice in most cardiology exercise labs, where formal consent is not obtained prior to a treadmill test.

All respondents have access to a cycle ergometer for testing. However, the majority (55\%) of departments only have access to a cycle ergometer, thereby limiting the range of patients who are physically able to perform the test. In addition to a cycle ergometer, eight (16\%) departments have access to a hand crank ergometer and seven (14\%) have access to a treadmill ergometer. One department (2\%) has access to bicycle, hand crank and treadmill ergometers.
A variety of sub-specialty patient groups are tested, as depicted in Figure 3. Other patient groups tested are pediatric cardiology, ICU follow-up and adult congenital heart disease follow-up (Figure 3).

The majority of respondents use the anaerobic threshold (AT) (90\%), Ve/VCO2 (71\%), peak VO2 (59\%), and $\mathrm{Ve} / \mathrm{VO} 2(31 \%)$ to risk-stratify patients. A variety of other CPET-derived parameters were also reported as being used for risk stratification. For example: onset of ischemia, oxygen pulse, heart rate response, blood pressure response, desaturation, ventilator limitation, $\mathrm{VO} 2 /$ work rate slope and $\mathrm{Ve} / \mathrm{VCO} 2$ slope.

The majority of respondents run their tests to the patients' symptom limited maximum (71\%). This provides evidence for the need for a clinician to be present during the test on the grounds of safety. Some terminate tests after the patient has exercised to their anaerobic threshold (14\%) or to their target peak VO2 (6\%). Other responses

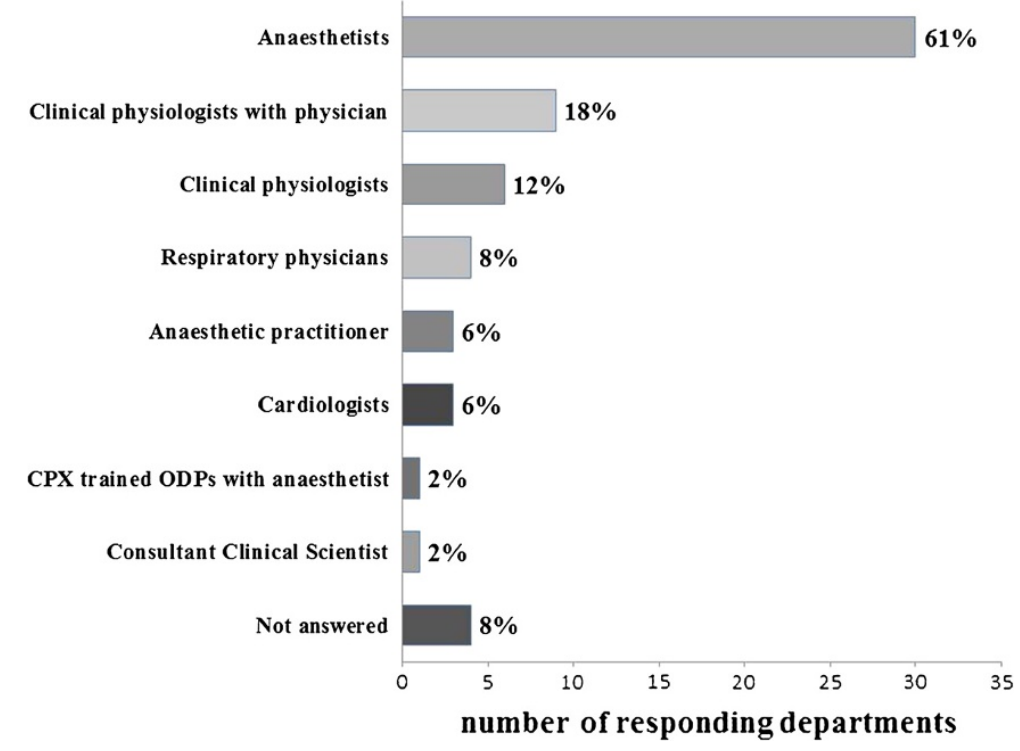

Figure 2 Who performs preoperative cardiopulmonary exercise testing? 


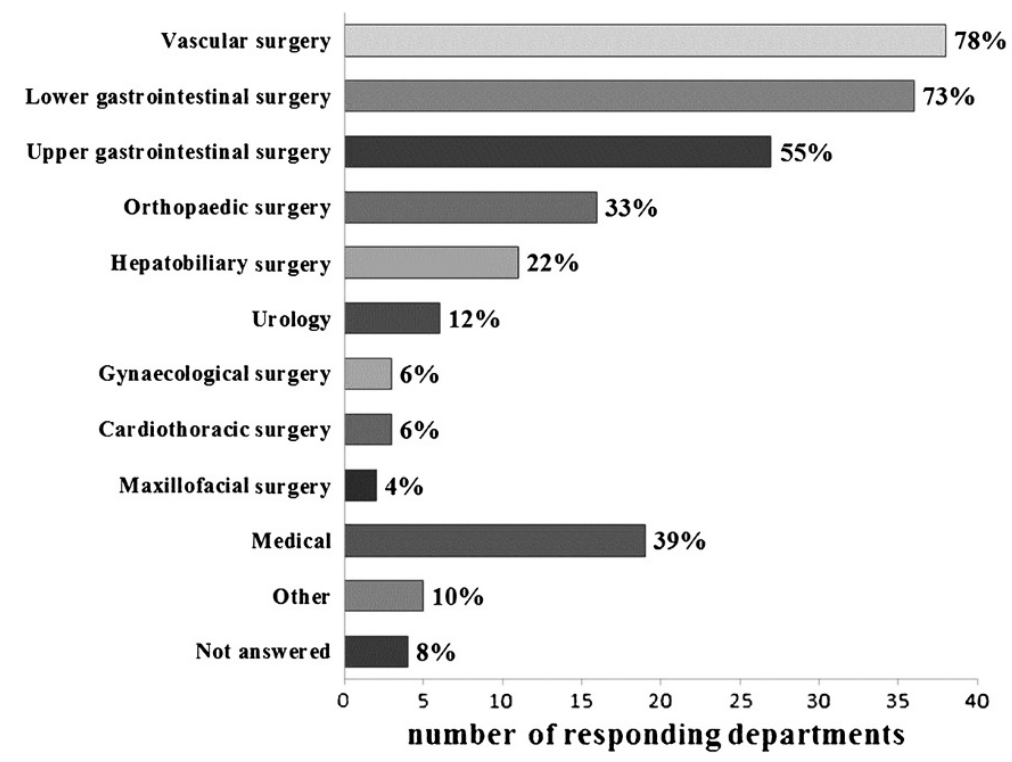

Figure 3 Which subspecialty groups are tested with PCPET?

include achieving predicted maximal heart rate and ischemic ECG changes.

The majority of respondents use the results for individual patient risk stratification and counseling (86\%) and to allocate patients to an appropriate level of post-operative care $(84 \%)$. Some departments use the results to determine the level of intraoperative monitoring (47\%). Other uses reported by respondents include: for clinical diagnostics, to modify surgical procedure (in support of funding applications for less invasive procedures in high risk cases) and to assess the need for pre-optimization.

The majority do not recommend cancellation of an individual case based on the CPET result (55\%). However, $33 \%$ of respondents do recommend cancellation of cases based on individual CPET results. There were many comments left for this question implying that the decision to cancel cases is more complex than this. The comments highlighted that CPET results advise risk and that the final decision lies with the patient, surgeon and, in some cases, the anesthetist. Some respondents felt strongly that the decision to cancel a case is not for the CPET clinic.

The median number of tests performed per clinic session was 3 (range 0.5-7.5, mean 3.2, mode 3, SD 7.3). The median number of PCPET clinic sessions per month was 6 (range 0.5-40, mean 8.4, mode 4, SD 1.5). Of the 53 departments identified by the telephone survey we received 49 responses. Of these 42 gave estimates regarding the output of testing services. If we substitute mode values (most conservative estimate) for missing data we estimate that over 15,000 PCETs are performed each year in England (Figure 4).

\section{Administrative and managerial aspects of PCPET services}

We were keen to find out how the administrative aspects of these clinics were managed. The questions ranged from organizing appointments to calibrating and validating the equipment and getting the reports to the referring clinician.

Appointments are arranged using written, formal appointment letters with an information leaflet in the majority of cases (76\%). Other methods include: by telephone $(8 \%)$ or written, formal appointment letters without information leaflet (8\%).

The PCPET equipment is owned either by anesthetic departments $(51 \%)$, respiratory departments $(20 \%)$, cardiology departments (10\%), clinical measurement/physiology departments (4\%), surgical departments $(2 \%)$ or clinical research facilities (2\%).

Respondents reported a variety of administrative support as part of their service infrastructure. These include: departmental secretary (39\%), pre-assessment clinic staff (27\%) and own secretary (10\%). Several respondents commented that administrative tasks are undertaken by clinicians. Nine services (18\%) have no administrative support.

Where anesthetists perform PCPET, the majority of sessions are considered as a clinical professional activity (51\%) in job plans. Some sessions are in supporting professional activity time (12\%), and others in the clinicians' own time (8\%).

The majority of tests performed are logged onto the hospitals' patient administration systems (67\%); $12 \%$ are not logged and $10 \%$ of respondents did not know if tests were logged. 


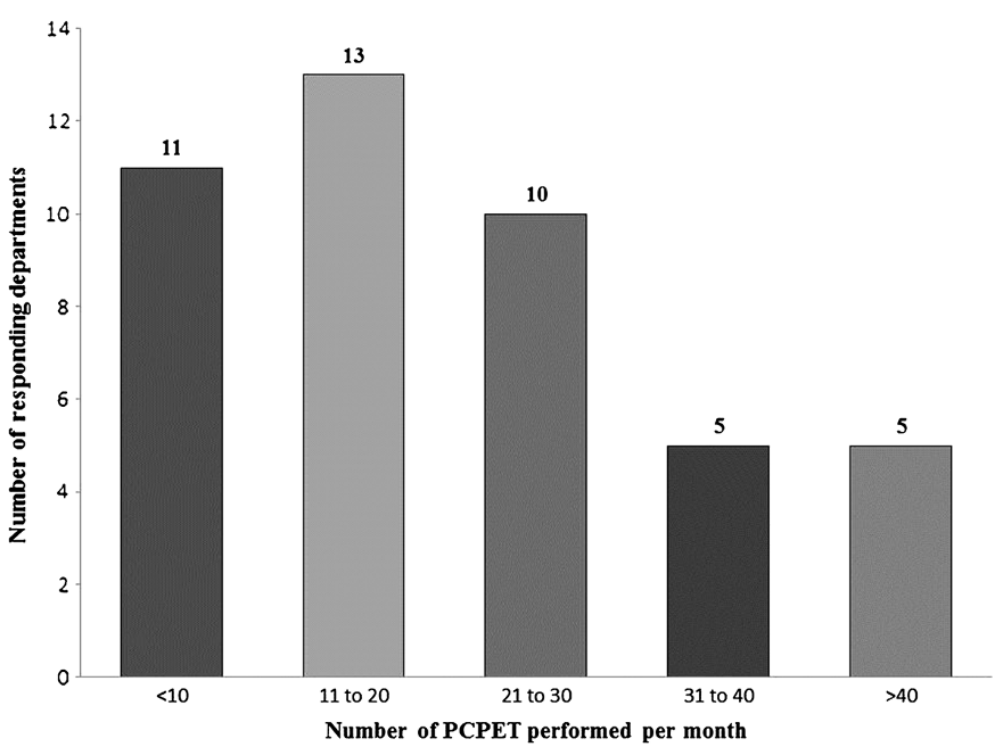

Figure 4 Peri-operative cardiopulmonary exercise testing output per month in England.

The primary care trust (PCT) is billed for the patients tested in only $37 \%$ of departments. No payment is received for testing in $31 \%$ of responding departments and $24 \%$ of respondents did not know if the PCT was billed.

\section{Discussion and conclusion}

We contacted $100 \%$ of individual anesthetic departments and identified $32 \%$ with CPET services and $4 \%$ in the process of setting up. In the 2008 survey Simpson et al. contacted $89 \%$ of trusts in England by telephone and received a $66 \%$ response rate when asking about the availability of CPET [1]. The higher response rate in our survey could be attributed to persistence with the telephone survey, the inclusion of a repeat telephone survey and accessibility of the online survey. The number of departments offering CPET has increased from $17 \%$ in 2008 to $32 \%$ in 2011 (while those in the process of setting up increased from $23 \%$ in 2008 to $36 \%$ in 2011). However, the 2008 survey may well have underestimated the availability of CPET due to their lower response rate. It is also unknown if any departments in the process of setting up a service were indeed successful. The 2008 survey was structured as nine open questions and sent as an email attachment. The authors commented that some responses were brief and lacked detail. In contrast our survey was online and included mostly multiplechoice questions with the opportunity for comment where appropriate. We believe this method increases the consistency of responses. However, we acknowledge that this style of questioning can be leading and may not collect some details of uncommon practice. The 2008 survey identified specific thresholds for parameters used for risk stratification, our survey did not. We have shown that the majority of patients tested have vascular, lower gastrointestinal or upper gastrointestinal symptoms. It appears that there is an extrapolation of the evidence to other unstudied surgical groups. The published evidence shows that different CPET parameters have variable predictive power in different sub-specialties [2-18]. Therefore, we believe extrapolating specific evidence to unstudied patient groups is not advisable.

We were surprised to find that the responses from departments without PCPET suggest that there is a desire to have these services in the majority of departments, but that the primary obstacles are financial. Some departments may not have the high-risk case load to justify their own service, although this was not commented on by any respondents. The results suggest that there is an interest in PCPET in the majority of anesthetic departments, but that the perceived obstacles are the financial implications of setting up such a service. However, the survey was targeted at those with an interest in preoperative assessment. This group may be more enthusiastic and not represent the views of all anesthetists.

Not all questions in the survey were compulsory. While this ensured a better response rate overall, we did receive responses with some unanswered questions. The median number of unanswered responses per question was four (8\%) (range 4-9, mean 4.9, SD 1.2) and this accounts for the shortfall in percentages of responses given in the results section.

There are a number of patient groups in whom PCPET does not have published outcome correlation data. These include patients requiring hepatobiliary, maxillofacial, gynecological or urological surgery. This highlights the importance of and potential for, further 
research into PCPET and post-operative outcomes, particularly in unstudied groups.

Departments use a wide variety of PCPET parameters to risk-stratify individual patients. Some parameters used are well established in the literature as good predictors of outcome (for example AT and Ve/VCO2) [2-11,14-18]. However, some studies suggest that AT is not a consistently strong predictor across all surgical groups $[12,13]$. Other parameters are also used to predict risk without published evidence, for example oxygen pulse. The variability of parameters used by respondents seems to reveal confusion over how results should be used to guide management. This is reflected in the inconsistencies of the reported predictive power of individual parameters published in the literature [2-18]. It would be interesting to know how many respondents use combinations of parameters or individual parameters alone (e.g. AT, $\mathrm{Ve} / \mathrm{VCO}_{2}$, peak $\mathrm{VO}_{2}$ or $\mathrm{Ve} /$ $\mathrm{VO}_{2}$ ) to risk-stratify patients.

Results are used for individual patient risk stratification and to allocate patients to the appropriate post-operative level of care. Some departments reported using results to determine the level of intra-operative monitoring. We are not aware of any specific evidence supporting this practice. One department reported using the results to support applications for funding for less invasive surgery (e.g. endovascular aortic aneurysm repair) in cases identified as high-risk cases by PCPET; again we are not aware of any evidence supporting this.

There appears to be controversy regarding the recommendation to cancel patients based on PCPET results. The majority of departments $(55 \%)$ do not recommend cancellation of individual cases solely on the basis of PCPET results. A third of departments do recommend cancellation on the basis of PCPET results. The comments suggest that those who cancel patients on the basis of test results do so in conjunction with surgical colleagues and other clinical patient information available to them.

We were surprised to see the estimated number of tests performed in England per year is in excess of 15,000 . Since we used modal values for missing data it is likely to be a conservative estimate. The numbers given in the survey responses do not necessarily take into account seasonal variations in output (e.g. bank holidays, annual leave and cancellations) and therefore may be overestimates. Even if we assume a month of testing is lost to these factors, the estimate is still in excess of 14,000 tests performed per year in England.

The number of tests, and the breadth of specialty groups in which testing is used, represents an exciting opportunity for collaborative research. Recent national attempts to establish communication and data sharing between departments will be a welcome step towards this goal (National PCPET Meeting, July 2011).
We were surprised to see that not all services are appropriately remunerated for the tests performed. Of respondents, $31 \%$ reported receiving no funding for tests from the PCT. Indeed a further $24 \%$ of respondents did not know if payment for tests was received from the PCT. We perceive this as being one of the key messages from the survey. Given that a significant proportion of respondents have indicated financial constraints as being the major hurdle in setting up these services, we were surprised to find that more than half the existing services were not generating income from their tests.

The survey has demonstrated the rapid growth of PCPET as a preoperative risk assessment tool in England. It has identified financial constraints as the main obstacle in setting up new services. This is despite clear evidence that there is significant national interest in having PCPET services available for the preoperative risk assessment of high-risk patients. We hope the results of the survey will add to the evidence presented in support of future attempts to establish new services.

\section{Additional files}

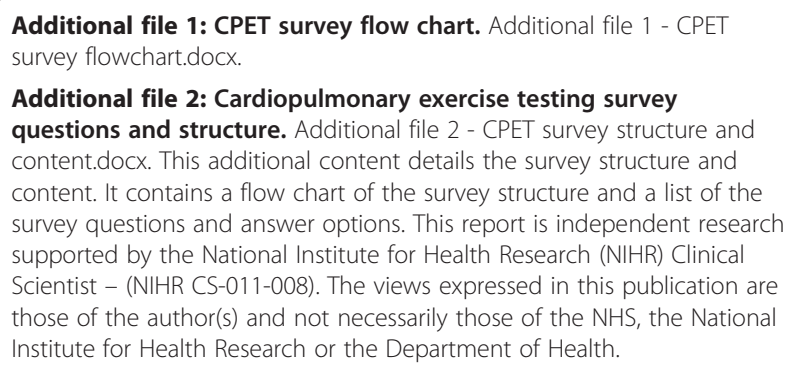

\section{Abbreviations}

AT: anaerobic threshold; CPET: cardiopulmonary exercise testing; ECG: electrocardiogram; NHS: National Health Service; PCPET: preoperative cardiopulmonary exercise testing; PCT: primary care trust; Ve/

VCO2: ventilatory equivalents for carbon dioxide; VeNO2: ventilatory equivalents for oxygen; VO2: oxygen consumption (liters per minute).

\section{Competing interests}

PJEH is on the editorial board of the Perioperative Medicine Journal.

\section{Authors' contributions}

SH co-wrote and designed the survey (with PKP), collected contact details and data, coordinated the data collection, interpreted data and drafted the manuscript. ELY collected contact details and data, independently validated data interpretation and helped in the draft of the manuscript. RS collected contact details and data, independently validated data interpretation and helped in the draft of the manuscript. PJEH independently validated data interpretation and helped in the draft of the manuscript. PKP conceived of the study, co-wrote and designed the survey with $\mathrm{SH}$, coordinated data collection, independently validated data interpretation, drafted the manuscript and supervised the project. All authors read and approved the final manuscript.

\section{Authors' information}

$\mathrm{SH}, \mathrm{RS}$ and ELY are anesthetic trainees at the St Georges School of Anaesthesia, London, UK. PH is an NIHR Clinical Scientist at the St George's Vascular institute and is currently working at the Queen Elizabeth Hospital, Adelaide, Australia. PKP is a consultant anesthetist and cardiopulmonary 
exercise testing lead at the Royal Surrey County Hospital, Guildford, Surrey, UK.

The results of this survey were presented by the authors as a poster and an oral presentation at the 4th National CPET Forum, the Institute of Education, London on 6 July 2011.

\section{Acknowledgments}

We would like all of the clinicians and support staff who responded to the surveys. In addition we thank Professor M Grocott and Dr W Fawcett for their invaluable advice on the writing of this manuscript.

\section{Author details}

${ }^{1}$ Department of Anaesthesia, Royal Surrey County Hospital, Guildford GU2 7XX, UK. ${ }^{2}$ Department of Anaesthesia, Mount Sinai Hospital, Toronto, Canada. ${ }^{3}$ St George's Vascular Institute, London, UK. ${ }^{4}$ The Queen Elizabeth Hospital, Adelaide, Australia.

Received: 22 May 2012 Accepted: 8 February 2013

Published: 25 February 2013

\section{References}

1. Carlisle J, Swart M: Mid-term survival after abdominal aortic aneurysm surgery predicted by cardiopulmonary exercise testing. Br J Surg 2007, 94(8):966-969.

2. Mancini DM, Eisen H, Kussmaul W, Mull R, Edmunds LH, Wilson JR: Value of peak exercise oxygen consumption for optimal timing of cardiac transplantation in ambulatory patients with heart failure. Circulation 1991, 83(3):778-786.

3. Older $P$, Hall A, Hader R: Cardiopulmonary exercise testing as a screening test for perioperative management of major surgery in the elderly. Chest 1999, 116(2):355-362.

4. Swart M, Carlisle JB: Case-controlled study of critical care or surgical ward care after elective open colorectal surgery. Br J Surg 2012, 99(2):295-299.

5. Wilson RJ, Davies S, Yates D, Redman J, Stone M: Impaired functional capacity is associated with all-cause mortality after major elective intraabdominal surgery. Br J Anaesth 2010, 105(3):297-303.

6. Snowden CP, Prentis JM, Anderson HL, Roberts DR, Randles D, Renton M, Manas DM: Submaximal cardiopulmonary exercise testing predicts complications and hospital length of stay in patients undergoing major elective surgery. Ann Surg 2010, 251(3):535-541.

7. Hightower CE, Riedel BJ, Feig BW, Morris GS, Ensor JE Jr, Woodruff VD, Daley-Norman MD, Sun XG: A pilot study evaluating predictors of postoperative outcomes after major abdominal surgery: physiological capacity compared with the ASA physical status classification system. Br J Anaesth 2010, 104(4):465-471.

8. McCullough PA, Gallagher MJ, Dejong AT, Sandberg KR, Trivax JE, Alexander D, Kasturi G, Jafri SM, Krause KR, Chengelis DL, Moy J, Franklin BA: Cardiorespiratory fitness and short-term complications after bariatric surgery. Chest 2006, 130(2):517-525.

9. Epstein SK, Freeman RB, Khayat A, Unterborn JN, Pratt DS, Kaplan MM: Aerobic capacity is associated with 100-day outcome after hepatic transplantation. Liver Transp/ 2004, 10(3):418-424.

10. Nugent AM, Riley M, Megarry J, O'Reilly MJ, MacMahon J, Lowry R: Cardiopulmonary exercise testing in the pre-operative assessment of patients for repair of abdominal aortic aneurysm. Ir J Med Sci 1998, 167(4):238-241Y.

11. Nagamatsu Y, Shima I, Yamana H, Fujita H, Shirouzu K, Ishitake T: Preoperative evaluation of cardiopulmonary reserve with the use of expired gas analysis during exercise testing in patients with squamous cell carcinoma of the thoracic esophagus. J Thorac Cardiovasc Surg 2001, 121(6):1064-1068.

12. Forshaw MJ, Strauss DC, Davies AR, Wilson D, Lams B, Pearce A, et al: Is cardiopulmonary exercise testing a useful test before esophagectomy? Ann Thorac Surg 2008, 85(1):294-299.

13. Junejo MA, Mason JM, Sheen AJ, Moore J, Foster P, Atkinson D, Parker MJ, Siriwardena AK: Cardiopulmonary exercise testing for preoperative risk assessment before hepatic resection. Br J Surg 2012, 99(8):1097-1104.

14. Ausania F, Snowden CP, Prentis JM, Holmes LR, Jaques BC, White SA, French JJ, Manas DM, Charnley RM: Effects of low cardiopulmonary reserve on pancreatic leak following pancreaticoduodenectomy. Br J Surg 2012, 99(9):1290-1294.
15. Prentis JM, Trenell MI, Jones DJ, Lees T, Clarke M, Snowden CP: Submaximal exercise testing predicts perioperative hospitalization after aortic aneurysm repair. J Vasc Surg 2012, 56:1564-1570.

16. Colson M, Baglin J, Bolsin S, Grocott MP: Cardiopulmonary exercise testing predicts 5 yr survival after major surgery. Br J Anaesth 2012. Epub ahead of print.

17. Hennis PJ, Meale PM, Hurst RA, O'Doherty AF, Otto J, Kuper M, Harper N, Sufi PA, Heath D, Montgomery HE, Grocott MP: Cardiopulmonary exercise testing predicts postoperative outcome in patients undergoing gastric bypass surgery. Br J Anaesth 2012, 109:566-571.

18. Society BT, Party IW: Guidelines on the selection of patients with lung cancer for surgery. Thorax 2001, 56(2):89-108.

19. Simpson JC, Sutton H, Grocott MPW: Cardiopulmonary exercise testing - a survey of current use in England. J Intensive Care Soc 2009, 10:275-278.

20. A-Z List of All NHS Acute (Hospital) Trusts in England. http://www.nhs.uk/ servicedirectories/pages/acutetrustlisting.aspx.

doi:10.1186/2047-0525-2-4

Cite this article as: Huddart et al.: Preoperative cardiopulmonary exercise testing in England - a national survey. Perioperative Medicine 2013 2:4.

\section{Submit your next manuscript to BioMed Central and take full advantage of:}

- Convenient online submission

- Thorough peer review

- No space constraints or color figure charges

- Immediate publication on acceptance

- Inclusion in PubMed, CAS, Scopus and Google Scholar

- Research which is freely available for redistribution 